\title{
Les atouts des coopératives : stabilité et expérience
} The cooperative advantage: Stability and experience Las ventajas de las cooperativas: estabilidad y experiencia

\section{Marius Chevallier}

Numéro 327, janvier 2013

URI : https://id.erudit.org/iderudit/1015150ar

DOI : https://doi.org/10.7202/1015150ar

Aller au sommaire du numéro

Éditeur(s)

Association Recma

ISSN

1626-1682 (imprimé)

2261-2599 (numérique)

Découvrir la revue

Citer cet article

Chevallier, M. (2013). Les atouts des coopératives : stabilité et expérience. Revue internationale de l'économie sociale, (327), 63-74.

https://doi.org/10.7202/1015150ar

\section{Résumé de l'article}

La stratégie contemporaine des entreprises et du mouvement coopératif et mutualiste consiste à affirmer que les coopératives et les mutuelles se caractérisent par des valeurs alternatives. Cette stratégie comporte des faiblesses, dans la mesure où il est difficile d'observer l'impact de telles valeurs dans les principaux secteurs d'activité de ces sociétés (industrie agroalimentaire, commerce de gros, banque, assurance). En revenant à leurs origines, on peut défendre l'hypothèse que les spécificités des coopératives résident plutôt dans des règles statutaires qui constituent des garde-fous à l'emprise des forces du marché. Alors que ce dernier valorise la réactivité et l'attractivité des travailleurs qualifiés, les coopératives et les mutuelles sont plutôt caractérisées par la stabilité et la capacité à créer et à exploiter des connaissances tacites et informelles adaptées à leur contexte spécifique. Or, cette logique marchande constitue le cadre d'analyse dominant, y compris pour les parties prenantes des coopératives : les individus ont « naturellement " tendance à puiser leurs solutions dans ce référentiel. Il apparaît donc nécessaire de s'en affranchir pour penser les coopératives sans renforcer les dynamiques de banalisation. 


\title{
LES ATOUTS DES COOPÉRATIVES: STABILITÉ ET EXPÉRIENCE
}

\author{
par Marius Chevallier*
}

* Geolab, université de Limoges. Mél.: marius.chevallier@unilim.fr.

La stratégie contemporaine des entreprises et du mouvement coopératif et mutualiste consiste à affirmer que les coopératives et les mutuelles se caractérisent par des valeurs alternatives. Cette stratégie comporte des faiblesses, dans la mesure où il est difficile d'observer l'impact de telles valeurs dans les principaux secteurs d'activité de ces sociétés (industrie agroalimentaire, commerce de gros, banque, assurance). En revenant à leurs origines, on peut défendre l'hypothèse que les spécificités des coopératives résident plutôt dans des règles statutaires qui constituent des garde-fous à l'emprise des forces du marché. Alors que ce dernier valorise la réactivité et l'attractivité des travailleurs qualifiés, les coopératives et les mutuelles sont plutôt caractérisées par la stabilité et la capacité à créer et à exploiter des connaissances tacites et informelles adaptées à leur contexte spécifique. Or, cette logique marchande constitue le cadre d'analyse dominant, y compris pour les parties prenantes des coopératives: les individus ont "naturellement" tendance à puiser leurs solutions dans ce référentiel. Il apparaît donc nécessaire de s'en affranchir pour penser les coopératives sans renforcer les dynamiques de banalisation.

(1) Loi d'Oppenheimer sur la banalisation ou la disparition des coopératives: une coopérative doit se transformer en entreprise à actionnariat ou disparaître. Oppenheimer F., 1914, L'économie pure et l'économie politique, préface de Charles Gide, Paris, M. Giard et E. Brière éditeurs. (NDLR.) es tendances à la banalisation des coopératives et des mutuelles ont fait l'objet d'une grande attention dans les entreprises et le mouvement coopératif et mutualiste, à l'instar de Jacques Moreau dans les années 80 (Chomel, Alix, 2005), comme chez les chercheurs depuis les travaux de Franz Oppenheimer au début du $\mathrm{xx}^{\mathrm{e}}$ siècle $^{(1)}$. Néanmoins, l'hypothèse que les coopératives et les mutuelles ont un fonctionnement différent de leurs concurrents reste très présente. Dans une perspective positive, cette différence se définirait par l'adhésion de leurs membres à des principes et à des valeurs spécifiques. Dans une optique plus négative, les coopératives et les mutuelles se caractériseraient plutôt par leur lenteur et un certain amateurisme résultant de leur faible attractivité des travailleurs qualifiés, de sorte qu'elles se retrouveraient condamnées à l'échec économique (disparition) ou politique (banalisation par l'importation de solutions issues des sociétés de capitaux). L'hypothèse défendue ici est que cette seconde optique paraît plus défendable que la première, mais qu'elle peut et doit être présentée comme un atout des coopératives et des mutuelles assurant la durabilité de ces dernières. La lenteur peut en effet être traduite positivement comme une capacité à créer de la stabilité dans un monde 
(2) La stabilité est particulièrement avantageuse en période de crise, mais elle pose plus de problèmes en période de crois sance, lorsque l'écart entre les coopératives et leurs concurrents devient trop important, à l'image des nombreuses disparitions de coopératives de consommateurs dans les années 70-80 (Brazda, Schediwy, 1989). Cette moindre cyclicité (Demoustier Colletis, 2012), si elle ne constitue pas toujours un avantage économique, se traduit par une temporalité économique plus conforme avec la temporalité humaine, d'où une économie plus " humaine». volatil $^{(2)}$ et l'amateurisme comme une faculté à développer et à exploiter des connaissances issues de l'expérience.

Dans une première section, nous rappelons la cohabitation de ces deux points de vue dans l'histoire des coopératives et des mutuelles (encadré 1), en donnant les raisons pour lesquelles il parait difficile aujourd'hui de se placer dans la perspective positive. Nous donnons ensuite une brève présentation du courant économique qui a développé la seconde optique, mais nous expliquons cependant qu'elle ne permet pas de conclure a priori à une inefficience des coopératives et des mutuelles: un renversement du cadre d'analyse permet de montrer que la stabilité et les connaissances issues de l'expérience constituent un atout pour ces entreprises. Dans une troisième section, nous confirmons alors la vraisemblance de cette deuxième optique, requalifiée en termes de stabilité-connaissances issues de l'expérience plutôt qu'en lenteur-amateurisme, en nous appuyant sur des données de l'enquête "Emploi » 2007 de l'Insee (encadré 2, en page suivante). Nous concluons en montrant qu'une stratégie de défense de la différence coopérative et mutualiste fondée sur la revendication de la stabilité et de la connaissance issue de l'expérience limiterait les risques de banalisation.

\section{Une double caractérisation historique des coopératives et des mutuelles}

Selon Claude Vienney (1980), les coopératives émergent parce que l'équilibre économique de certains acteurs est déstabilisé par l'évolution des normes dans leurs secteurs d'activité: "c'est le cas lorsqu'il s'agit de combinaisons qui, au regard des règles des nouveaux agents dominants, ne sont pas à la fois "productives et rentables" (Vienney, 1980, p. 71-72). "Certaines catégories de producteurs, pour certaines activités "perturbées"

\section{Encadré 1 Champ d'analyse}

Dans cet article, nous traitons ensemble les coopératives et les mutuelles. D'une part, elles partagent deux caractéristiques que sont l'organisation démocratique et la lucrativité limitée, deux caractéristiques largement mobilisées dans la littérature économique traitant de ces organisations. D'autre part, les coopératives de certains secteurs d'activité sont appelées mutuelles dans d'autres pays et inversement (notamment dans le domaine de la banque et de l'assurance). Par la suite, le vocable " coopératives " peut donc valoir pour " coopératives et mutuelles " afin d'alléger la rédaction. Les coopératives de travailleurs (Scop en France) ne constituant qu'une petite partie du monde coopératif, l'analyse vaut d'abord pour les entreprises dont les membres sont les usagers et les fournisseurs (coopératives agricoles, coopératives de commerçants, coopératives de crédit, mutuelles d'assurance santé et de dommages), même si nous avons également mobilisé une littérature sur les coopératives de travailleurs. 


\section{Encadré 2 Méthodologie}

Cette recherche se caractérise paradoxalement par la mobilisation d'une littérature économique largement défavorable aux coopératives pour mieux défendre la durabilité du modèle coopératif. La comparaison de 728 emplois coopératifs et de 26000 emplois en sociétés de capitaux à partir de l'enquête «Emploi » 2007 de l'Insee vient toutefois confirmer que les coopératives sont moins réactives et moins capables de mobiliser une connaissance formelle que les sociétés de capitaux. Ce travail bibliographique et empirique est alors complété par un travail analytique permettant de distinguer les résultats fiables de la conclusion ad hoc selon laquelle les coopératives et les mutuelles ne seraient pas viables. $\mathrm{Au}$ contraire, la version positive de ces résultats - la capacité à créer de la stabilité et à développer et mobiliser des connaissances issues de l'expérience - tend à conclure que les coopératives et les mutuelles constituent un modèle durable d'entreprise. Nous suggérons un appui sur une littérature institutionnaliste, pour conforter cette piste de recherche.

(3) Voire de les modifier en profondeur, sil'on considère que certaines coopératives et mutuelles ont été à l'origine de normes qui ont fini par s'imposer sur leurs marchés, telles que les coopératives de consommateurs pour une baisse générale des prix et du développement de normes sanitaires strictes ou encore les mutuelles de travailleurs au $\mathrm{XIX}$ siècle à I'origine du système de sécurité sociale. Je remercieles rapporteurs pour cette remarque. par les capitalistes-entrepreneurs [...], peuvent trouver dans le groupement de leurs fonctions d'achat de moyens de production, de mobilisation de leurs épargnes et de distribution de crédit, d'approvisionnement en denrées de consommation courante les moyens de sortir relativement des situations de dépendance qui résultent de la généralisation du capitalisme industriel. "

\section{Se libérer des normes économiques dominantes}

A la fin du XIX ${ }^{\mathrm{e}}$ siècle, les coopératives de consommateurs sont nées pour permettre un approvisionnement en denrées alimentaires à des coûts plus bas que ceux proposés par les commerçants, considérant que ces coûts élevés les maintenaient dans la pauvreté. De même, certaines mutuelles d'assurance dommages ont été créées en réaction aux cotisations excessives imposées à des catégories socioprofessionnelles, ou encore des agriculteurs groupés sont parvenus à montrer qu'il était possible de vendre plus cher leur production. D'autres motifs que le prix ont pu être moteurs. Par exemple, les banques d'affaires étaient relativement fermées aux petites et moyennes entreprises. Or, l'accès aux capitaux devenant essentiel pour limiter la dépendance de ces dernières à des acteurs économiques concentrés, elles ont commencé à se regrouper sur des bases sectorielles et territoriales pour mutualiser leurs ressources en caisses locales, qui deviendront les banques populaires. Dans le secteur du bâtiment, des artisans se sont également regroupés pour avoir un accès direct à la clientèle alors qu'ils étaient devenus les sous-traitants d'entreprises générales ayant standardisé la construction de pavillons. Les coopératives et les mutuelles se créent donc d'abord en réaction à une norme économique. A l'origine, il y a cette idée selon laquelle le regroupement des acteurs leur permet de tempérer ${ }^{(3)}$, de mettre à distance, l'emprise des normes économiques qui structurent leurs secteurs. 
L'enjeu est loin d'être seulement économique, car ces déséquilibres peuvent menacer la survie même d'un groupe social, regroupant en général des acteurs économiques qui appartiennent à un même secteur ou à un même territoire. Ainsi, on retrouve généralement une dimension revendicative, souvent relayée par les syndicats, avec l'expression de valeurs telles que l'entreprise à taille humaine pour les petites et moyennes entreprises et les artisans, la solidarité rurale pour les coopératives agricoles, la défense du monde ouvrier pour les coopératives de consommateurs, etc. A l'origine des coopératives, il y a donc aussi cette idée quelles peuvent contribuer à défendre des valeurs.

\section{Des valeurs alternatives?}

Les entreprises et les mouvements coopératifs et mutualistes français contemporains insistent plutôt sur la deuxième dimension de l'origine à travers la question des valeurs. Ces entreprises seraient pleinement dans le marché, mais auraient des valeurs alternatives. Il semble difficile, pourtant, de défendre l'idée selon laquelle les $10 \%$ de l'emploi national (selon le Conseil national des chambres régionales d'économie sociale, CNCres, 2010) que représentent les coopératives, les mutuelles, les associations et les fondations puissent s'inscrire dans cette optique de valeurs alternatives qui auraient un impact significatif sur le comportement des individus. Rappelons, pour ce qui est des coopératives et des mutuelles, que plus de $90 \%$ du volume d'activité est réalisé par de grandes entreprises dans l'industrie agroalimentaire (InVivo, Sodiaal, Terrena, Triskalia, etc.), le commerce de gros (Leclerc, System U, Bigmat, Intersport, ainsi que Astera et Welcoop en pharmacie, etc.) et la finance (banque et assurance) [tableau 1].

\section{Tableau 1}

Principaux secteurs d'activité des coopératives et des mutuelles en France

\begin{tabular}{|c|c|c|c|}
\hline & $\begin{array}{c}\text { Nature } \\
\text { des membres }\end{array}$ & $\begin{array}{c}\text { Volume d'affaires } \\
\text { (en milliards d'euros) }\end{array}$ & Poids dans le secteur \\
\hline $\begin{array}{c}\text { Commerce } \\
\text { de gros }\end{array}$ & $\begin{array}{c}\text { Commerçants } \\
\text { détaillants }\end{array}$ & 124 (CA) & $\begin{array}{c}28 \% \text { des commerçants } \\
\text { détaillants }\end{array}$ \\
\hline $\begin{array}{c}\text { Finance } \\
\text { (banque, assurance) }\end{array}$ & $\begin{array}{c}\text { Personnes physiques } \\
\text { ou personnes } \\
\text { morales }\end{array}$ & $\begin{array}{c}110 \text { (produit net } \\
\text { bancaire pour les banques } \\
\text { et cotisations } \\
\text { pour les mutuelles) }\end{array}$ & $\begin{array}{c}60 \% \text { de l'activité } \\
\text { de la banque de détail, } \\
56 \% \text { des cotisations } \\
\text { en assurance santé }\end{array}$ \\
\hline $\begin{array}{c}\text { Agriculture et industrie } \\
\text { agroalimentaire }\end{array}$ & Agriculteurs & $82(\mathrm{CA})$ & $75 \%$ des agriculteurs \\
\hline $\begin{array}{c}\text { Sociétés coopératives } \\
\text { et participatives } \\
\text { (Scop) }\end{array}$ & $\begin{array}{c}\text { Travailleurs } \\
\text { salariés }\end{array}$ & $3,5(\mathrm{CA})$ & $\begin{array}{c}\text { Moins de 2 \% de l'activité } \\
\text { bâtiment, principal } \\
\text { secteur d'activité des Scop }\end{array}$ \\
\hline
\end{tabular}

Sources: données tirées du panorama sectoriel des entreprises coopératives publié en 2010 par CoopFR, de la Mutualité française pour les $56 \%$ des cotisations dans l'assurance santé, de la CGScop pour le poids du secteur du bâtiment dans l'ensemble du chiffre d'affaires des Scop (65\%) et de la Fédération française du bâtiment pour le chiffre d'affaires de ce domaine en 2010 (125 milliards). 
(4) Nous avons assisté à une dizaine d'assemblées générales en 2010 dans des coopératives agricoles, bancaires et des mutuelles, ainsi qu'à diverses manifestations de la Fédération du commerce associé. Caire (2010) note également qu'il est illusoire d'identifier l'expression de valeurs alternatives dans les assemblées générales auxquelles il a assisté dans le secteur bancaire.
La participation à des temps institutionnels d'entreprises de ces secteurs en France (assemblées générales ${ }^{(4)}$, regroupements fédéraux) semble plutôt indiquer que les normes régissant les coopératives et les mutuelles sont identiques à celles régissant les comportements de leurs concurrents, même si des valeurs particulières sont ponctuellement observées (Weber, 2011).

Lobjectif est donc, ici, de revenir à la première des deux origines des coopératives et des mutuelles: la capacité à s'émanciper partiellement des normes qui structurent les secteurs dans lesquels elles sont insérées. L'hypothèse est qu'elles se caractérisent par leur capacité à tempérer l'emprise des normes de réactivité et d'attractivité des travailleurs qualifiés structurant le champ économique aujourd'hui (Chevallier, 2011).

\section{Défauts de réactivité et d'attractivité des coopératives}

(5) Cent quarante articles ont été analysés sur les années 1970 à 2009 à partir des bases de données Econlit et Business Source Premier. Celles-ci recensent des revues qui respectent un certain nombre de critères académiques, surreprésentant notamment la version standard de l'économie (Favereau, 1989; Colletis, 2010). L'école française des coopératives y est relativement absente. En 2009, la Recma n'était pas encore recensée.

(6) Alchian et Demsetz (1972), Jensen et Meckling (1976), Fama et Jensen (1983), Furubotn et Pejovich (1972), Grossman et Hart (1986), Vitaliano (1983), Williamson (1975). Ces auteurs des années 70-80 sont encore dominants parmi les références bibliographiques aujourd'hui (Jossa, 2009).

(7) Ces auteurs partagent notamment des croyances non discutées telles que l'efficience des mécanismes de marché capables de conduire à un équilibre généra optimal ou la rationalité des acteurs capables de prendre des décisions individuelles optimales à partir des informations dont ils disposent (Colletis, 2010).
Une revue de la littérature à partir des mots clés "cooperativ" et "mutual " ${ }^{(5)}$ permet de constater que les bibliographies accordent une place prépondérante aux auteurs de la nouvelle économie institutionnelle ${ }^{(6)}$ fortement influencés par le cadre de pensée néoclassique ${ }^{(7)}$ autour de Jensen et Meckling (1976), avec un fort consensus sur l'identification de caractéristiques unanimement considérées comme des déficiences. On distingue cependant deux groupes d'auteurs. Les défenseurs de la société de capitaux - et donc des droits de propriété des apporteurs de capitaux comme condition de la performance des entreprises - entendent montrer que les coopératives sont une forme inefficace qui ne devrait pas survivre durablement. Les défenseurs des coopératives soutiennent, eux, qu'il est possible de corriger ces déficiences. La position défendue est que cette littérature permet effectivement de caractériser avec pertinence les spécificités coopératives, mais en ajoutant immédiatement, grâce à un cadre de pensée différent du cadre néoclassique, que ces caractéristiques ne sont pas des déficiences et qu'elles doivent donc être défendues plutôt que corrigées.

\section{De la « lenteur " coopérative}

On peut résumer les caractéristiques des coopératives identifiées par les économistes en deux grandes catégories (pour plus de détails sur cette revue de littérature, voir Chevallier, 2011). La plus analysée dans la littérature relève de ce qui est négativement considéré comme une certaine lenteur (un problème de réactivité, une source de rigidité). Ainsi, les auteurs montrent que les coopératives connaissent des obstacles à une prise de décision rapide, en raison du pluralisme des objectifs et du poids des démarches statutaires. Ensuite, une fois les décisions prises, les coopératives souffrent d'une difficulté à accéder aux ressources nécessaires à l'application de ces décisions à cause de leur manque de flexibilité, caractéristique essentielle des marchés. 
(8) Une analyse plus récente sur des coopératives agricoles francaises montre que ces dernières sont caractérisées par une plus forte longévité (Carrère etal., 2011). (9) On retrouve d'ailleurs ici un paradoxe déjà relevé dans la littérature sur les personnes âgées (Sala, Guéret-Talon, 2007) et, pourtant, on ne constate pas de différence globale de productivité (Volkoff, 2000). Cette littérature connexe peut donc constituer une voie de recherche pour comprendre la performance des coopératives.
Compte tenu du principe de double qualité (Draperi, 2007), les coopératives ne peuvent pas facilement mettre un terme aux relations qui les lient, car leurs membres sont à la fois des investisseurs et des consommateurs, des fournisseurs ou des travailleurs.

Cela renforce les problèmes de réactivité des coopératives, mais, surtout, on trouve ici leur deuxième caractéristique majeure: une difficulté d'attractivité des individus les plus qualifiés (autant au niveau des travailleurs que des coopérateurs). La littérature empirique permet de constater que les coopératives sont en effet en retard dans l'introduction des innovations se diffusant dans leurs secteurs en matière de marketing, de financement, de gestion des ressources humaines, etc. Les dirigeants salariés des coopératives se plaignent d'ailleurs souvent de se retrouver fréquemment dans le deuxième train, à la suite de leurs concurrents.

\section{Et de leur présumé manque d'attractivité}

Pourtant, ces déficiences ne semblent pas se traduire par une moindre efficience, quels que soient les indicateurs mobilisés. Trois synthèses (Doucouliagos, 1997, pour les labor-managed firms; Sexton et Iskow, 1993, pour les coopératives agricoles ${ }^{(8)}$; Fortin et Leclerc, 2011, pour les coopératives bancaires) montrent, en moyenne, qu'il n'est pas possible de donner de conclusion univoque sur la comparaison des efficiences des entreprises selon leurs statuts juridiques ${ }^{(9)}$. Si les résultats observés s'avèrent différents des prévisions des économistes, c'est que leur cadre d'analyse néoclassique régi par le marché est inadapté à la compréhension des coopératives. Les auteurs de cette économie néoinstitutionnelle tendent à réduire l'entreprise à un nœud de contrats, par opposition à l'approche institutionnaliste, qui, elle, reconnaît dans l'entreprise une institution à part entière, irréductible à la somme de ses parties prenantes (Demoustier, Colletis, 2012). La littérature institutionnaliste semble mieux adaptée à la compréhension de la durabilité des coopératives et des mutuelles (Borzaga et al., 2011; Demoustier, 2012). Les performances des entreprises résident largement dans la capacité à mobiliser des compétences tacites et informelles et des routines (Mangolte, 1997), c'est-à-dire des compétences développées progressivement au sein de l'entreprise dans un contexte de stabilité plutôt que possédées par des individus hautement qualifiés recrutés sur un marché $\mathrm{du}$ travail très concurrentiel. Certaines entreprises peuvent assurer leurs performances grâce à leur capacité à attirer des individus très efficaces, mais ces derniers, très demandés, sont en général également très mobiles et leur bref passage rend difficile la coordination interne avec l'ensemble des salariés. En revanche, d'autres entreprises sont moins attractives sur le marché du travail, mais la stabilité de leurs équipes et de l'ensemble des parties prenantes leur permet de développer une connaissance fine des problématiques, des compétences adaptées au contexte spécifique de l'entreprise, mais difficilement transférables à d'autres contextes (Colletis, Pecqueur, 1993). Dans la section suivante, nous confirmons 
les résultats de la littérature économique néoclassique, mais nous abandonnons le vocabulaire négatif de défaut de réactivité et d'attractivité pour parler de stabilité et de connaissances issues de l'expérience.

\section{Les coopératives entre stabilité et connaissances issues de l'expérience}

(10) Quatre-vingts pour cent des emplois sont répartis dans trois secteurs sur les seize qui constituent la nomenclature d'activités française (NAF): cette enquête révèle que $56,9 \%$ des emplois sont dans la finance et l'assurance, $15,9 \%$ dans le commerce et $6,3 \%$ dans l'industrie agroalimentaire, ce qui est conforme à la répartition mesurée par l'Insee la même année.

(11) Etudiant l'impact des conventions collectives, Gerlach et Stephan (2008) trouvent des différences de deux années.
Les données de l'enquête «Emploi » 2007 de l'Insee viennent renforcer les résultats empiriques présentés et réinterprétés dans la partie précédente. Cette enquête contient des informations sur 728 emplois coopératifs et 25599 emplois de sociétés de capitaux. La méthode consiste à analyser les différences des moyennes (test T, ou test de Student) pour plusieurs variables entre la population des emplois en coopératives et celle des emplois en sociétés de capitaux.

\section{Un emploi plus durable dans les coopératives}

Tous secteurs confondus ${ }^{(10)}$, la moyenne de l'ancienneté des emplois dans les coopératives est de 13,9 ans, contre 9,8 ans pour les sociétés de capitaux. On retrouve ces différences dans deux des trois principaux secteurs où les coopératives sont présentes (tableau 2).

Les différences de deux à trois ans ${ }^{(11)}$ sont significatives selon un test $\mathrm{T}$ au seuil de $0,1 \%$ pour l'industrie agroalimentaire et le commerce de gros, mais pas pour l'assurance et la finance. L'impact du statut coopératif sur la stabilité de l'emploi est d'autant plus significatif que l'on constate que les femmes, dont l'ancienneté est en moyenne inférieure à celle des hommes, sont surreprésentées dans les emplois coopératifs (54\%, contre $37 \%$ dans les sociétés de capitaux), ce qui devrait tirer l'ancienneté moyenne de ces derniers vers le bas.

L'ancienneté moyenne supérieure des emplois dans les coopératives est un indicateur d'une culture de la stabilité. Celui-ci est complété par une analyse de la nature des contrats. La stabilité de l'emploi coopératif est confirmée par un tableau de contingence croisant la nature des contrats avec celle des entreprises et permettant de constater

\section{Tableau 2}

Ancienneté moyenne en fonction du statut et du secteur

\begin{tabular}{|l|c|c|}
\hline & Sociétés de capitaux & Entreprises coopératives \\
\hline Industrie agroalimentaire & 10,2 & 12,9 \\
\hline Commerce de gros & 10,1 & 13 \\
\hline Finance, assurance & 14 & 15 \\
\hline
\end{tabular}

Source: enquête "Emploi" 2007, Insee. 


\section{Tableau 3}

\section{Nombre de CDI et autres contrats}

\begin{tabular}{|l|l|c|c|}
\cline { 2 - 4 } \multicolumn{2}{c|}{ Tableau de contingence } & CDI & Autres contrats \\
\hline \multirow{2}{*}{ Sociétés de capitaux } & Constaté & 17777 & 2532 \\
\cline { 2 - 4 } & Attendu & 17801 & 2509 \\
\hline \multirow{2}{*}{ Entreprises coopératives } & Constaté & 642 & 63 \\
\cline { 2 - 4 } & Attendu & 618 & 87 \\
\hline
\end{tabular}

Source: enquête "Emploi" 2007, Insee. La valeur "attendue " est celle que l'on trouverait si la part des CDI dans l'emploi coopératif et leur part dans l'emploi des sociétés de capitaux étaient les mêmes. On compare cette valeur théorique à celle constatée.

(12) Le test V de Cramer permet de montrer que la population des coopératives est significativement différente de celle des sociétés de capitaux au seuil de $1 \%$.

(13) L'analyse statistique devra donc être complétée d'enquêtes de terrain pour comprendre comment cette culture de la stabilité se transmet. que les coopératives sont surreprésentées dans les contrats à durée indéterminée $(\mathrm{CDI})$ et sous-représentées pour tous les autres contrats $(\text { tableau } 3)^{(12)}$.

\section{Un moindre niveau de qualification}

Enfin, le niveau de qualification est moindre dans les coopératives (tableau 4). Or, plus il est important, plus les travailleurs se situent sur un marché du travail gouverné par une forte primeà la mobilité (Colletis, 2008), qui apparait contradictoire avec le développement et la mobilisation de connaissances situées car issues de l'expérience des individus.

Rappelons, enfin, que les salariés ne sont sociétaires de leur coopérative uniquement dans le cas minoritaire des coopératives de travailleurs. Dans celles-ci, on comprend aisément que les travailleurs aient plus d'ancienneté que dans les sociétés de capitaux, mais les coopératives étudiées ici concernent essentiellement des salariés qui ne sont pas toujours sociétaires. Le fait que l'ancienneté de l'emploi y soit néanmoins significativement différente de celle des sociétés de capitaux suggère qu'il existe dans les coopératives une culture de la stabilité qui se transmet à l'ensemble des acteurs, au-delà des seules contraintes statutaires ${ }^{(13)}$.

\section{Tableau 4}

Niveau de qualification des cadres en fonction du statut de l'entreprise

\begin{tabular}{|c|c|c|c|c|c|}
\hline $\begin{array}{c}\text { Qualification } \\
\text { des cadres }\end{array}$ & $\begin{array}{c}\text { Supérieur } \\
\text { à bac + 2 }\end{array}$ & Bac + 2 & Bac & CAP-BEP & $\begin{array}{c}\text { Sans } \\
\text { diplôme }\end{array}$ \\
\hline Sociétés de capitaux & $56,70 \%$ & $17,50 \%$ & $12,70 \%$ & $7,20 \%$ & $5,90 \%$ \\
\hline Coopératives & $35,50 \%$ & $27,10 \%$ & $21,30 \%$ & $9,70 \%$ & $6,40 \%$ \\
\hline
\end{tabular}

Source: enquête "Emploi " 2007, Insee, étude réalisée sur une population de 3804 cadres travaillant dans des entreprises à actionnariat et 155 dans des coopératives. 


\section{Conclusion: changer de paradigme pour limiter les risques de banalisation}

(14) La correction du défaut de réactivité ne mène cependant pas nécessairement à la banalisation. Les coopératives peuvent présenter certains atouts pour internaliser la flexibilité, comme c'est notamment le cas dans les coopératives de travailleurs, dont les salariés sociétaires peuvent jouer sur leur niveau de revenus pour maintenir l'emploi en période de crise (voir par exemple Prades, 2005). Je remercie les rapporteurs pour cette remarque.
Dans l'imaginaire dominant qui valorise avant tout la réactivité et l'attractivité, les caractéristiques coopératives sont frappées d'un $a$ priori défavorable. Pour améliorer leur fonctionnement, il est proposé de corriger ces spécificités et donc de banaliser ces entreprises ${ }^{(14)}$. Cet imaginaire dominant tend à nier la coopérative dans ce qui la caractérise et à faire de la société de capitaux, structurellement plus conforme aux principes de réactivité et d'attractivité, l'horizon ultime de la coopérative. Dans certains cas, cela va jusqu’à la démutualisation (l'abandon du statut coopératif), notamment dans l'assurance et la finance, au sein de pays tels que l'Australie, le Royaume-Uni, la Belgique, les Etats-Unis, l'Afrique du Sud, le Canada, la NouvelleZélande et la Suède (Davis, 2001), tandis que d'autres phénomènes de démutualisation semblent inéluctables dans le secteur de la finance en Espagne (Carrasco, 2006).

Les évolutions des normes légales qui régissent les comportements dans les coopératives sont souvent interprétées comme visant essentiellement à alléger les contraintes qui pèsent sur la réactivité et l'attractivité de ces entreprises. Ces évolutions participent ainsi de la banalisation des coopératives (Monzon-Campos, Zevi, 1995). Chomel et Vienney (1995) notent que, dans le cas français, une série d'ordonnances vient autoriser dans les années 60 les coopératives à instaurer des différences de traitement entre leurs membres pour éviter que les meilleurs clients et fournisseurs partent à la concurrence. De 1970 à 1990, ce mouvement s'amplifie: il devient possible d'accepter une partie de clients non coopérateurs dans les mutuelles d'assurance et les banques coopératives (loi de 1972 pour les coopératives agricoles, loi de 1983 pour les coopératives d'artisans). Cela permet à la fois d'accepter des investisseurs non coopérateurs et, pour répondre au problème de l'attractivité des coopératives sur le marché des capitaux, de leur garantir une meilleure rémunération. Enfin, la loi de modernisation de 1992 permet aux associés non coopérateurs de disposer de voix proportionnellement au capital détenu, dans la limite de $35 \%$, et autorise de puiser en partie dans les réserves impartageables pour réévaluer les parts sociales. On a également vu se multiplier les créations de filiale échappant aux règles coopératives, renforçant la création de valeur pour l'actionnaire plutôt que pour l'usager (Mauget, Forestier, 2000) et permettant une meilleure adéquation à la flexibilité des relations marchandes ou encore un principe de sélection et de rémunération différenciée dans les coopératives agricoles (Jarrige, Touzard, 2001). Ainsi, de nombreuses normes issues des sociétés de capitaux sont introduites dans les entreprises coopératives, venant diluer leur modèle et le conformer à la représentation dominante. 
Dans le contexte contemporain de multiplication de crises économiques et financières, certaines voix non congruentes à la représentation dominante en termes de réactivité et d'attractivité peuvent plus légitimement s'exprimer. C'est l'occasion de construire une représentation alternative plus conforme au modèle coopératif. Pour cela, la littérature institutionnaliste semble être un précieux allié, afin de réaliser à quel point les dimensions tacites et informelles des relations humaines sont essentielles à la performance des entreprises et s'inscrivent dans un temps long. Il s'agirait alors de réaffirmer les caractéristiques coopératives plutôt que de les « corriger " conformément au modèle des sociétés de capitaux. Ceux qui cherchent à renouveler et à renforcer le rôle des coopérateurs dans leur entreprise coopérative et mutualiste (Birchall, Simmons, 2004; Richez-Battesti, 2008) acquerraient ainsi une source supplémentaire de légitimité. 


\section{Bibliographie}

Alchian A., Demsetz H., 1972, « Production, information costs and economic organization ", American Economic Review, n 62 , p. $777-795$.

Birchall J., Simmons R., 2004, " The involvement of members in the governance of large-scale cooperative and mutual businesses: A formative evaluation of the cooperative group ", Review of social economy, vol. 62, $\mathrm{n}^{\circ} 4$.

Borzaga C., Depedri S., Tortia E., 2011, "Diversité des organisations dans les économies de marché, rôle des coopératives et des entreprises sociales: plaidoyer en faveur d'un pluralisme économique ", Recma, n 321.

Brazda J., Schediwy R. (dir.), 1989, Consumer cooperatives in a changing world, International cooperative alliance, Genève.

Caire G., 2010, "De la démocratie locale dans certaines banques coopératives ", Recma, $\mathrm{n}^{\circ} 316$.

Carrasco I., 2006, « The global financial environment and spanish cooperative banking ", International Advances in economic research, vol. 10, n ${ }^{\circ}$, p. 265-272.

Carrère M., Joly I., Rousselière D., 2011, "De la longévité coopérative, une étude de la survie des coopératives agricoles françaises", Recma, n' 320.

Chevallier M., 2011, « Les coopératives entre rationalité formelle et rationalité située ", thèse de doctorat en sciences économiques, université de Toulouse.

Chomel A., Alix N., 2005, Pour une économie sociale sans rivages: Jacques Moreau (1927-2004), L'Harmattan, coll. " Les cahiers de l'économie sociale ", n ${ }^{\circ} 5$.

Chomel A., Vienney C., 1995, « Evolution des principes et des règles des organisations coopératives en France ", in Monzon-Campos, Zevi (1995), p. 117-154.
CNCres, 2010, Panorama national de l'ESS en France et dans les régions, www.cncres.org. Colletis G., 2008, "Financiarisation, mondialisation et nouveau rapport salarial ", in Colletis G., Paulré B. (dir.), Les nouveaux horizons du capitalisme: pouvoirs, valeurs et temps, Paris, Economica.

Colletis G., 2010, « Pour un débat sur les représentations en économie ", Esprit, décembre.

Colletis G., Pecqueur B., 1993, « Intégration des espaces et quasi-intégration des firmes; vers de nouvelles logiques productives?", Revue d'économie régionale et urbaine, $\mathrm{n}^{\circ} 3$, p. 490-507.

Davis K., 2001, "Credit union governance and survival of the cooperative form ", Journal of financial services research, vol. 19, $n^{\circ} 2$, p. 197-210.

Demoustier D., 2012, «ESS: de l'approche entrepreneuriale à une perspective institutionnaliste ", Recma, n 325.

Demoustier D., Colletis G., 2012, "L'économie sociale et solidaire face à la crise: simple résistance ou participation au changement? ", Recma, n 325.

Doucouliagos C., 1997, "The comparative efficiency and productivity of labor-managed and capital-managed firms ", Review of radical political economics, vol. 29, n 2, p. 45-69. Draperi J.-F., 2007, Comprendre l'économie sociale, fondements et enjeux, Dunod.

Fama E., Jensen M., 1983, "Separation of ownership and control ", Journal of law and economics, vol. 26, n 2, p. 301-325.

Favereau O., 1989, "Marchés internes, marchés externes ", Revue économique, $\mathrm{n}^{\circ} 2$, p. 273-328.

Fortin M., Leclerc A., 2011, « L'efficience des coopératives de services financiers ", Annals of public and cooperative economics, vol. 82, $\mathrm{n}^{\circ} 1$, p. 45-62. 
Furubotn E., Pejovich S., 1972, «Property rights and economic theory: A survey of recent literature ", Journal of economic literature, vol. 10, no 4, p. 1137-1162.

Gerlach K., Stephan G., 2008, «A note on job tenure and collective contracts ", Review of labor economics and industrial relations, vol. 22, $\mathrm{n}^{\circ}$ 1, p. 167-183.

Grossman S., Hart O., 1986, "The costs and benefits of ownership: A theory of vertical and lateral intergration ", Journal of political economy, vol. $94, n^{\circ} 4$, p. 691-719.

Jarrige F., Touzard J., 2001, «Les mutations de l'organisation coopérative à travers l'évolution de ses règles: la rémunération de la vendange dans les caves coopératives du Midi ", Recma, n 280.

Jensen M., Meckling W., 1976, "Theory of the firm: Managerial behavior, agency costs and ownership structure ", Journal of financial economics, $n^{\circ} 3$, p. 303-360.

Jossa B., 2009, "Alchian and Demsetz's critique of the cooperative firm thirty years after ", Metroeconomica, vol. 60, n 4 , p. 686-714.

Mangolte P., 1997, «La dynamique des connaissances tacites et articulées: une approche socio-cognitive ", Economie appliquée, L (2), 105-34.

Mauget R., Forrestier M., 2000, «De la coopérative au groupe coopératif agroalimentaire: de la valeur pour l'usager à la valeur pour l'actionnaire? ", Recma, n' 278.

Monzon-Campos J., Zevi A. (dir), 1995, Coopératives, marchés, principes coopératifs, De Boeck.
Prades J., 2005, « L'énigme de Mondragon, comprendre le sens de l'expérience ", Recma, $\mathrm{n}^{\circ} 296$.

Richez-Battesti N., 2008, "Gouvernance coopérative et sociétariat: une reconquête inachevée? Une illustration par les banques coopératives en France ", in Draperi J.-F. (coord.), Défis coopératifs: alimentation, crédit, démocratie, développement, L'Harmattan, coll. "Les cahiers de l'économie sociale ", n 6 .

Sala F., Guéret-Talon L., 2007, Pérennité au travail, âge, bouleversements et performance, Chronique sociale.

Sexton R., Iskow J., 1993, "What do know about the economic efficiency of cooperatives? An evaluative survey ", Journal of agricultural cooperation, $\mathrm{n}^{\circ} 8$, p. 15-27.

Vienney C., 1980, Socio-économie des organisations coopératives, Ciem, Paris.

Vitaliano P., 1983, "Cooperative enterprise: An alternative conceptual basis for analyzing a complex institution ", American Journal of agricultural economics, $n^{\circ}$ 65, p. 1078-1083.

Volkoff S., Molinié A., Jolivet A., 2000, Efficaces à tous âges? Vieillissement démographique et activités de travail, La Documentation française, dossier du centre d'études et d'emploi, n' 16 .

Weber J., 2011, « Au-delà du contrat, la mobilisation du concept de valeur de lien dans la relation sociétaire-mutuelle d'assurance : le cas de la Macif ", Recma, no 321.

Williamson O., 1975, Markets and hierarchies, analysis and antitrust implications: $A$ study in the economics of internal organization, The Free Press. 